MPIKG Public Access

Author Manuscript

Published in final edited form as:

Antonietti, M., \& Savateev, A. (2018). Splitting Water by Electrochemistry and Artificial Photosynthesis: Excellent Science but a Nightmare of Translation? The Chemical Record, 18, 969-972. doi:10.1002/tcr.201700062.

\title{
Splitting water by electrochemistry and artificial photosynthesis: excellent science but a nightmare of translation?
}

\author{
Markus Antonietti*, Alexandr Savateev
}

Keywords: artificial photosynthesis; value chemicals; translation; alternative targets

This article may be used for non-commercial purposes in accordance with Wiley Terms and Conditions for Self-Archiving. 


\title{
Splitting water by electrochemistry and artificial photosynthesis: excellent science but a nightmare of translation?
}

\author{
Markus Antonietti*, Alexandr Savateev \\ Max Planck Institute of Colloids and Interfaces, Colloid Chemistry, Research Campus Golm, Am Mühlenberg 1, \\ 14476 Potsdam (Germany).
}

\begin{abstract}
Water splitting to its elements, either by electrochemistry or by solar light, is among the most covered areas in nanostructured functional materials. This personal account article analyzes potential downstream translation problems and reviews alternative chemistries with a potential higher return. Liberation of oxygen for accepting the holes is a kinetically demanding half reaction afflicted with kinetic hindrances and high overpotentials, while at the same time no marketable value is created (atmospheric oxygen is free to use). In spite of exciting science created, application in real industrial set-ups is currently impossible, and possible funding promises to contribute to a sustainable society become a debt difficult to return. We discuss possible alternative targets of (photo)electrochemistry as entry points where chemical value products and technical oxidants are created, with partially greater ease, lower losses, and higher benefits.
\end{abstract}

\section{Introduction}

The artificial leaf, but materials for man-made photosynthesis as such are in the recent focus of modern materials chemistry. The possibility to turn solar energy directly into chemical fuel (see, for instance, refs $1-3$ ) is inspired by Nature and thereby appealing already by that. Similar arguments can be made for the alternative use of stranded electrical energy in devices based on electrochemical water splitting (for some of the best articles, see refs $4-7$ chosen from about 5000 available publications). Also here, science and engineering develops with great enthusiasm water oxidation and water reduction catalyst, which for us are partly masterpieces for creativity and excellence. But: many of us (including me) promise a better world to justify extended funding, and we must be serious on how much these promises have a real chance for translation, i.e. can we keep them?

At the current standing of the field, the as generated hydrogen would be simply not competitive with fossil alternatives, as shale gas and other methane resources seem to be available in high volume. Assuming a 2 Volt electrolyzer and $1 \mathrm{kWh}$ of green electric energy to cost around $0.1 €$, the $\mathrm{kg}$ of green hydrogen comes for $5.37 € / \mathrm{kg}$, energy content only, without investments or additional installments and workforce. This is to be compared with current fossil methane prices of about 120 $160 € / t$, which can be turned " $\mathrm{CO}_{2}$-emmission free" by thermal decomposition into fossil hydrogen 
for about $0.8 € / \mathrm{kg}$, again no running costs calculated. It is obvious that only most gracious public subsidies can close this gap, and there are certainly better uses for the tax-payers money than locking-in a specific technology for many years. In other words: no company will invest in even a perfect technology in this direction as the ecologic benefits are much less than the added economic expenses. The argument is not getting better when using the as-generated electrons for $\mathrm{CO}_{2}$ reduction instead of hydrogen liberation: it is the generated value per electron in all those chains which questions the process, and as long it is good value to make hydrogen from methane, the opposite will turn into a grand loss operation. Other prominent reviews (better: personal accounts) in this direction argue that the price of solar energy will further decline, in curves similar to the increase of computer power (8). We personally doubt this (as energy is no data and related to matter), but even following a more idealistic approach and assuming solar energy for free, solar energy valorization is simply restricted by the solar constant. The solar influx of radiation in Germany can be translated, when using a $10 \%$ efficient overall solar water splitting device, into $2 \mathrm{~kg}$ hydrogen per $\mathrm{m}^{2}$ and year, i.e. solar energy is rather "diluted". With this return, can we fabricate and run $1 \mathrm{~m}^{2}$ of a rather complicated device, full of nano- and microstructured functional metals and rare elements? It is clear that we have about $20 € / \mathrm{m}^{2}$ for that, assuming very long lifetimes and hypothetical turnover numbers for the catalysts exceeding $10^{11}$. There is no escape from the reality of those numbers: the cheaper the energy, the lower the possible investments for the functional device, and indeed only high energy prizes enable innovative technology.

Facing these realities, we could declare now our current scientific grand plans and promises as chimeras, wishful dreams, and there is a risk that the opponents of a green energy change might do that. We could be also slightly ashamed about our own naivety, as we have ignored the world of economy as well as very basic natural principles and constants. As the involved science is however so rich and so powerful, defeat is not intended. The current preposition of this article is the reframing of the problem, however now including value and economy as (non-scientific) side conditions. The approach is to define alternative targets for artificial photosynthesis/green electrochemistry with higher chemical value gain, and in the following, some better targets than hydrogen and oxygen are reviewed.

\section{Alternative cathode reactions}

Reduction of dioxygen to $\mathrm{OH}^{-}$in a 4-electron process is the base of all electrochemical fuel-to-energy processes, say in fuel cells, and development of improved ORR (oxygen reduction reaction) catalysts will stay a key theme in our community for many years. For the inverse process, i.e. molecule generation from energy, this search can be complemented by exploring the 2-electron transfer instead of the usually discussed 4-electron transfer. Then, the well traded product hydroperoxide 
(instead of oxygen) is obtained as a rather save aqueous solution. Along the previously discussed cost lines, a $\mathrm{kg}$ of $\mathrm{H}_{2} \mathrm{O}_{2}$ can be made photo-electrochemically for $0.32 € / \mathrm{kg}$, to be compared with the current "fossil" market price of about $1.5 € / \mathrm{kg}$, the latter value however being a consumer price. With metal catalysts, this electrochemistry is practically impossible, as most ORR agents also decompose $\mathrm{H}_{2} \mathrm{O}_{2}$, but appropriately chosen nanostructured, heterogeneous carbocatalyst with high specific surface area are indeed very selective and only catalyze the two electron reduction of oxygen, but not the two competing side paths (9). The real advantage of simple electrochemistry is here that the peroxide can be generated in a decentral fashion in small doses "on demand", e.g. for disinfection and bleaching purposes, essentially avoiding the necessity of transport and storage of such a dangerous good. Here, we note that peroxides, perborates and percarbonates constitute for instance a major fraction of laundry formulations. Electrochemistry could become as such a part of daily household machines.

Another highly relevant and valuable target is to "coin" the hydrogen into an activated species useful for other reactions, say for enzymes. Here, for instance $\operatorname{NADH}$ made from $\operatorname{NAD}^{+}(10,11)$ can drive complex enzymatic and biological cascades. Making NADH, enzymatic chains can be "fed" by photoor electrochemistry. Using the combination of photochemical NADH generation and enzymatic post conversion, a special biomimetic nanostructured carbon nitride catalyst to drive the chiral reduction of aldehydes was described (11), showing up to $60 \%$ apparent quantum yield and being overall very efficient. It is worth mentioning that NADH generation does not rely on a cocatalyst, as the involved CT- supported electron transfer followed by a proton transfer is by orders of magnitude more efficient than the formation of a covalent $\mathrm{H}-\mathrm{H}$ bond.

A comparable synthetic target is the photogeneration of dihydropyridines or Hantzsch-Esters (12). This reaction came rather unexpected and was enabled by special high surface area polyheptazineimides. Again, an active organic hydride carrier is created with unexpected ease. In this case, the stored hydride can be accessed by organocatalysis (13), and again the handling of hydrogen for substrate hydrogenation could be avoided. Both the NADH generation as well as the dihydropyridine synthesis work "all-organic", i.e. without the use of platinum or other noble metals. Of course these applications are, when compared to fuels, niche markets, but the coupling of artificial photosynthesis and biotechnology is inspiring and might result in some next generation concepts and thoughts directed to high value products.

\section{Alternative anode reactions}

The oxidation of water to molecular oxygen is known to be the bottleneck of all the mentioned lightto-fuel or electron-to-fuel technologies. This reaction is a well analyzed, complicated 4-electron oxidation cascade, it is coupled to spin transfer problem (as dioxygen usually comes in the triplet 
state), it is therefore very sluggish, and even the best nanostructured hybrid electrocatalysts rely on overpotentials of around 0.20 Volt -0.30 Volt to realize semi-meaningful production rates, that is at least $20 \%$ of the invested electric energy is already lost from the beginning for this half-cell reaction. The whole problem is even worse for artificial photosynthesis, as in this case the adjustment of rates is usually not directly in the hands of the scientists, and an elaborated system engineering is necessary to match the two half-cell rates to realize a sufficiently high internal quantum yield (IQY) within the device. This was nicely elaborated by Tarascon and Yang (14). In most practical cases, the oxidation half reaction is simply too slow, thus restricting the overall cell and hydrogen productivity.

This is also why most research on artificial photosynthesis is restricting itself only the reductive half reaction, replacing the dioxygen liberation by the oxidation of a sacrificial agent, and we have to admit that we do that also in our own work (e.g. 15, 16). Of course this is a highly restrictive simplification: the spin forbidden 4-electron transfer is replaced by a more simple one electron transfer usually coming with much lower overpotentials, the rate of which can be even adjusted by substrate concentration and $\mathrm{pH}$. Again a point is reached where we have to question our simplifications: are these really necessary to improve access to research funding?

A part of the answer falls back to the discussion above: the liberation of oxygen in such reactions is not only energy inefficient and sluggish, but also non-economic, and just because biology has decided for this path, it is not a must for a chemical process to do so. It is maybe too trivial, but a process which creates for $2 \mathrm{~g}$ of a product (hydrogen) $32 \mathrm{~g}$ of a non-marketable side product (oxygen) is usually not continued in technology, and any industrial engineer or business person would be forced to create added value also from the oxidation side.

Here, modern green chemistry usually "forgets" that THE running megascale electrolysis is the chloralkali electrolysis (17), which creates not only one product, but three, namely chlorine, sodiumhydroxide, and hydrogen. This is obviously good business, as no less than 65 Million tons of chlorine are produced per year, world-wide. This reaction family is indeed is a worthy global target: the energy input of chloralkali electrolysis is comparable to cementum industry, and a possible energy reduction is accountable in a global balance. In addition, the oxidation of aqueous chloride to the chlorine-radical is a simple and fast process, has comparably low overpotentials, and the standard redox potential of chlorine (1.36 Volt) is very close to water oxidation (1.23 Volt). Considering real life conditions and overpotentials, it is indeed practically more simple to liberate chlorine instead of oxygen.

Interestingly, simple chlorine (or similar anions) is not systematically addressed, but initial observations were - presumably first accidentally - already made in artificial photosynthesis 
experiments $(18,19,20)$. When water splitting is performed with model sea water or in a phosphate buffer, the efficiencies start to rise strongly, and the IQY partly exceeds 0.5 , i.e. the overall processes (the at least threefold convolute of light-to charge conversion, hydrogen elimination, and the photooxidation reaction) are run already not too far from perfection in very simple stirred beaker experiments. One of the papers even identified some details of the chlorine hole shuttle process (19), but put it into a typical Z-scheme context.

it is clear that the previous rate-determining step of four electron dioxygen generation is then remarkably simplified: the surface bound photocatalyst hole $(+)$ can in principle directly react with the anions bound locally in the Helmholtz layer at the interface of the nanostructured catalyst, and phosphate can be similarly oxidized to perphosphates (21). Chloride is oxidized under those (alkaline) conditions to hypochlorite, $\mathrm{ClO}^{-}$, even in the absence of a photooxidation catalyst. This bypasses even a secondary problem of oxygen liberation, namely the generally low stability of such photooxidation catalysts.

Chlorine chemistry is very classical but nowadays hardly known to the laymen, it is "forgotten" chemistry, so to say. Sodiumhypochlorite is a marketable product, valuable for disinfection, and once acidified, it spontaneously generates (singlet) oxygen (22). That way, man-made photosynthesis would open the chance to generate such chemicals again in a decentral fashion, also under rural conditions of the developing world, avoiding transport and storage of these partly dangerous products to a large extent. A solar cell and some functional electrocatalyst nanostructure would then be enough to disinfect water.

If the generation of oxygen is still the final goal, one can still generate it, far from the photo- or electrocatalyst, at any time with all the care it needs by acidification of the stored hypochlorite solution. It is a good question why Nature never explored chlorine chemistry and chose the more complicated direct oxygen release. Possibly, the involved oxidation potentials are simply too high for the used biochemical machinery, but this restriction does not apply for technological solutions.

Of course there are many other attractive targets in organic chemistry where oxidation reactions could contribute with a second product to the overall value chain. For instance, glycerol could be turned into the much more valuable dihydroxyacetone (23), or benzene could be turned into phenol, then phenol could be further coupled to polyphenols, but this is worth a more synthetic review article. As compared to inorganic oxidants, all those applications are however niche applications, highly valuable, but too far from the ordinary consumer. 


\section{Conclusion and Outlook}

The apparently most simple model reaction for uphill photocatalysis or electrocatalysis, the splitting of water, is to our opinion a target reaction which is -in spite of its apparent simplicity- unnecessarily complicated while generating too little value at the same time. This makes the translation into real societal applications very difficult. The involved science is indeed very refined and highly appealing, but should not be sold against better knowledge in the name of a global application target. The general arguments also hold true also when the solar or renewable hydrogen is used for $\mathrm{CO}_{2}$ fixation, and $\mathrm{CO}_{2}$-to-fuel conversion can be considered as on the long run uneconomic window dressing. Any feasibility analysis has to consider the whole chain and has to integrate through the whole usage cascade. Even when enormous funding is provided to save classical industrial approaches (as steel manufacturing or combustion engines), science should keep its objectivity and resist arguments which are mainly political or even psychological. Chemical $\mathrm{CO} 2$ fixation will only contribute to the real climate or energy change when green energy is much cheaper than fossil energy as such, and then we should of course first stop to burn fossils in power plants.

Chemistry is able to generalize uphill photosynthesis and can create solutions and devices beyond the biological master patterns for broader applications. Instead of using only the product of reduction in such processes, we should develop catalysts and catalytic cascades where both the oxidation and the reduction product and potentially also other side products represent a value, such as historically exemplified with the chloralkali electrolysis. And: we see more options, here. Interestingly, along those paths the use of complicated reduction and especially oxidation cocatalysts can be omitted. Inorganic peroxo-derivatives or also biological hydride transfer agents can be made without any cocatalyst, but can nevertheless be turned into oxygen and hydrogen whenever wanted, as so well-known from for laundry oxidants in washing and disinfection processes.

From a conceptual point, it is interesting to ask why in artificial photosynthesis we are so much bound to oxygen generation as the path for getting rid of the hole equivalents, and the answer is of course the idol of biology. Nature however had potentially no other choice (after the primary consumption of sulfides and low valent iron species), being bound to evolution of previously developed solutions and cascades. In Biomimetic Material Science, it is however possible to generalize a principle beyond an employed molecular or materials bas. Leaving the protein space and its stability restrictions and going for instance to modern carbon-nitrogen nanostructures (24) indeed enables to access more simple, more scalable photoredox systems with broader chemical applications. 


\section{Acknowledgements}

The authors want to thank the Max Planck Society for continued support. MA also appreciates the funding and the many discussions within the German Excellence Cluster UNICAT, Berlin.

\section{References}

[1] Water splitting-biosynthetic system with $\mathrm{CO} 2$ reduction efficiencies exceeding photosynthesis

Chong Liu1,2, ${ }^{*}$, Brendan C. Colón3, ${ }^{*}$, Marika Ziesack3, Pamela A. Silver3,†, Daniel G. Nocera1, ${ }^{\dagger}$ Science 03 Jun 2016: Vol. 352, Issue 6290, pp. 1210-1213

[2] The Artificial Leaf By: Nocera, Daniel G. ACCOUNTS OF CHEMICAL RESEARCH Volume: 45 Issue: 5 Pages: 767-776 Published: MAY 2012 Artificial Leaf

[3] Water-Splitting Catalysis and Solar Fuel Devices: Artificial Leaves on the Move Joya, KS (Joya, Khurram Saleem)[ 1,2,3 ] ; Joya, YF (Joya, Yasir F.)[ 5 ] ; Ocakoglu, K (Ocakoglu, Kasim)[ 6 ] ; van de Krol, R (van de Krol, Roel)[ 4 ] ANGEWANDTE CHEMIE-INTERNATIONAL EDITION Volume: 52 Issue: 40 Pages: 10426-10437 Published: SEP 272013

[4] Biomimetic molecular water splitting catalysts for hydrogen generation By:Joya, KS (Joya, Khurram Saleem)[ 1,2 ] ; de Groot, HJM (de Groot, Huub J. M.)[ 1 ] INTERNATIONAL JOURNAL OF HYDROGEN ENERGY Volume: 37 Pages: 8787-8799 : MAY 2012

[5] Benchmarking Heterogeneous Electrocatalysts for the Oxygen Evolution Reaction By:McCrory, CCL (McCrory, Charles C. L.)[ 1 ] ; Jung, SH (Jung, Suho)[ 1 ] ; Peters, JC (Peters, Jonas C.)[ 1,2 ] ; Jaramillo, TF (Jaramillo, Thomas F.)[ 1,3 ] JOURNAL OF THE AMERICAN CHEMICAL SOCIETY : 135 Pages: 16977-16987 : NOV 132013

[6] The Mechanism of Water Oxidation: From Electrolysis via Homogeneous to Biological Catalysis

By:Dau, H (Dau, Holger)[ 1 ] ; Limberg, C (Limberg, Christian)[ 2 ] ; Reier, T (Reier, Tobias)[ 3 ] ; Risch, M (Risch, Marcel)[ 1 ] ; Roggan, S (Roggan, Stefan)[ 2 ] ; Strasser, P (Strasser, Peter)[ 3 ] CHEMCATCHEM : 2 Pages: 724-761 Published: JUL 2010

[7] Strongly Coupled Inorganic/Nanocarbon Hybrid Materials for Advanced Electrocatalysis By:Liang, YY (Liang, Yongye)[ 1,2 ] ; Li, YG (Li, Yanguang)[ 1 ] ; Wang, HL (Wang, Hailiang)[ 1 ] ; Dai, HJ (Dai, Hongjie)[ 1 ] JOURNAL OF THE AMERICAN CHEMICAL SOCIETY 135 pages: 2013-2036 Published: FEB 132013 
[8] The path towards sustainable energy By: Chu, Steven; Cui, Yi; Liu, Nian NATURE MATERIALS Volume: 16 Issue: 1 Pages: 16-22 Published: JAN 2017

[9] Mesoporous Nitrogen-Doped Carbon for the Electrocatalytic Synthesis of Hydrogen Peroxide By:Fellinger, TP (Fellinger, Tim-Patrick)[ 1 ] ; Hasche, F (Hasche, Frederic)[ 2 ] ; Strasser, P (Strasser, Peter)[ 2 ] ; Antonietti, M (Antonietti, Markus)[1 ] JOURNAL OF THE AMERICAN CHEMICAL SOCIETY Volume: 134 Pages: 4072-4075 Published: MAR 72012

[10] PHOTOREDUCTION OF NAD+ TO NADH IN SEMICONDUCTOR DISPERSIONS CUENDET, P; GRATZEL, M

PHOTOCHEMISTRY AND PHOTOBIOLOGY Volume: 39 Pages: 609-612 Published: 1984

[11] Bio-inspired NADH regeneration by carbon nitride photocatalysis using diatom templates By: Liu, Jian; Antonietti, Markus ENERGY \& ENVIRONMENTAL SCIENCE Volume: 6 Issue: 5 Pages: 1486-1493 Published: MAY 2013

[12] Highly crystalline poly(heptazine imides) by mechanochemical synthesis for photooxidation of various organic substrates using an intriguing electron acceptor - Elemental sulfur By:Savateev, A (Savateev, Aleksandr)[ 1 ] ; Dontsova, D (Dontsova, Dariya)[ 1 ] ; Kurpil, B (Kurpil, Bogdan)[ 1 ] ; Antonietti, M (Antonietti, Markus)[ 1 ] JOURNAL OF CATALYSIS Volume: 350 Pages: 203-211 JUN 2017

[13] Recent developments in asymmetric transfer hydrogenation with Hantzsch esters: A biomimetic approach By: You, Shu-Li CHEMISTRY-AN ASIAN JOURNAL Volume: 2 Issue: 7 Pages: 820-827 Published: 2007

[14] Towards systems materials engineering By: Yang, Peidong; Tarascon, Jean-Marie NATURE MATERIALS Volume: 11 Issue: 7 Pages: 560-563 Published: JUL 2012

[15] Polymer Semiconductors for Artificial Photosynthesis: Hydrogen Evolution by Mesoporous Graphitic Carbon Nitride with Visible Light By:Wang, XC (Wang, Xinchen)[ 1,2 ] ; Maeda, K (Maeda, Kazuhiko)[ 3 ] ; Chen, XF (Chen, Xiufang)[ 2 ] ; Takanabe, K (Takanabe, Kazuhiro)[ 3 ] ; Domen, K (Domen, Kazunari)[ 3 ] ; Hou, YD (Hou, Yidong)[ 2 ] ; Fu, XZ (Fu, Xianzhi)[ 2 ] ; Antonietti, M (Antonietti, Markus)[ 1 ] JOURNAL OF THE AMERICAN CHEMICAL SOCIETY Volume: 131 Pages: 1680+ Published: FEB 112009

[16] e.g.: Carbon-doped BN nanosheets for metal-free photoredox catalysis :Huang, CJ (Huang, Caijin)[ 1 ] ; Chen, C (Chen, Cheng)[ 1 ] ; Zhang, MW (Zhang, Mingwen)[ 1 ] ; Lin, LH (Lin, Lihua)[ 1 ] ; Ye, XX (Ye, Xinxin)[ 1 ] ; Lin, S (Lin, Sen) [ 1 ] ; Antonietti, M (Antonietti, Markus)[ 2 ] ; Wang, XC (Wang, Xinchen)[ 1 ]

NATURE COMMUNICATIONS 6 , 7698, JUL 2015

[17] http://www.essentialchemicalindustry.org/chemicals/chlorine.html 
[18] Tri-s-triazine-Based Crystalline Graphitic Carbon Nitrides for Highly Efficient Hydrogen Evolution Photocatalysis, Lihua Lin, Honghui Ou, Yongfan Zhang, and Xinchen Wang , ACS Catal. 2016, 6, 3921-3931

[19] . Li, J.-X.; Ye, C.; Li, X.-B.; Li, Z.-J.; Gao, X.-W.; Chen, B.; Tung, C.-H.; Wu, L.-Z. Adv. Mater. 2017, 1606009.

[20] Optimizing Optical Absorption, Exciton Dissociation, and Charge Transfer of a Polymeric Carbon Nitride for Solar Hydrogen Production with an AQY of 57\% Guigang Zhang,[a] Zhi-An Lan,[b] Lihua Lin,[b] Aleksandr Savateev,[a] Tobias Heil,[a] Vasiliki Papaefthimiou,[c] Xinchen Wang*[b] and Markus Antonietti*[ Angew. Chem. Int. Ed. DOI: 10.1002/anie.201706870. http://onlinelibrary.wiley.com/doi/10.1002/anie.201706870/full

[21] Electrochemical Synthesis of Inorganic Compounds: A Bibliography, Zoltan Nagy Springer Science \& Business Media, 2013

[22] AHSAN ULLAH KHAN AND MiChAel KASHA, Proc. Natl. Acad. Sci. USA Vol. 91, pp. 1236212364, December 1994)

[23] Selective catalytic oxidation of glycerol: perspectives for high value chemicals By:Katryniok, B (Katryniok, Benjamin)[ 1,2,3 ] ; Kimura, H (Kimura, Hiroshi)[ 4 ] ; Skrzynska, E (Skrzynska, Elzbieta)[ 5 ] ; Girardon, JS (Girardon, Jean-Sebastien)[ 2,3 ] ; Fongarland, P (Fongarland, Pascal)[ 1,2,3 ] ; Capron, M (Capron, Mickael)[ 2,3 ] ; Ducoulombier, R (Ducoulombier, Remy)[ 2,3 ] ; Mimura, N (Mimura, Naoki)[ 6 ] ; Paul, S (Paul, Sebastien)[ 1,2,3 ] ; Dumeignil, F (Dumeignil, Franck)[ 2,3,7 ] GREEN CHEMISTRY 13 : 1960-1979: 2011

[24] Toward a green way for the chemical production of supported graphenes using porous solids Cristina Ruiz-García, Margarita Darder, Pilar Aranda and Eduardo Ruiz-Hitzky *J. Mater. Chem. A, 2014, 2, 2009-2017 